\title{
Prevalence of Suspected Hereditary Cancer Syndromes and Germline Mutations Among a Diverse Cohort of Probands Reporting a Family History of Prostate Cancer: Toward Informing Cascade Testing for Men.
}

Thenappan Chandrasekar

Thomas Jefferson University

Laura Gross

Thomas Jefferson University

Leonard G. Gomella

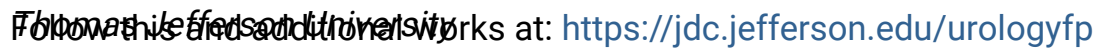

SrakrE.fHegartyology Commons, and the Urology Commons

thertasisgefteramoveriltarsitw access to this document benefits you

joon Yau Leong

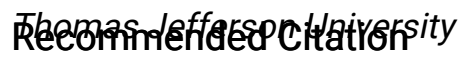

Chandrasekar, Thenappan; Gross, Laura; Gomella, Leonard G.; Hegarty, Sarah E.; Leong, Joon

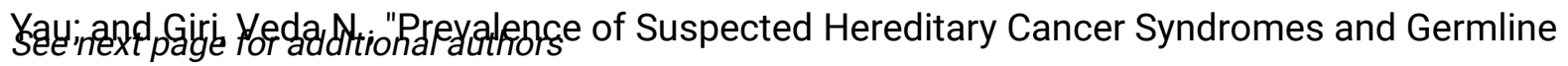
Mutations Among a Diverse Cohort of Probands Reporting a Family History of Prostate Cancer: Toward Informing Cascade Testing for Men." (2019). Department of Urology Faculty Papers.

Paper 50.

https://jdc.jefferson.edu/urologyfp/50

This Article is brought to you for free and open access by the Jefferson Digital Commons. The Jefferson Digital Commons is a service of Thomas Jefferson University's Center for Teaching and Learning (CTL). The Commons is a showcase for Jefferson books and journals, peer-reviewed scholarly publications, unique historical collections from the University archives, and teaching tools. The Jefferson Digital Commons allows researchers and interested readers anywhere in the world to learn about and keep up to date with Jefferson scholarship. This article has been accepted for inclusion in Department of Urology Faculty Papers by an authorized administrator of the Jefferson Digital Commons. For more information, please contact: JeffersonDigitalCommons@jefferson.edu. 
Authors

Thenappan Chandrasekar, Laura Gross, Leonard G. Gomella, Sarah E. Hegarty, Joon Yau Leong, and Veda N. Giri 
Title: Prevalence of suspected hereditary cancer syndromes and germline mutations among a diverse cohort of probands reporting a family history of prostate cancer: Towards informing cascade testing for men

Running Title: Germline mutations in African American families with prostate cancer

Authors: Thenappan Chandrasekar, MD, ${ }^{1}$ Laura Gross, BA, ${ }^{2}$ Leonard G. Gomella, MD, ${ }^{1}$ Sarah E. Hegarty, MPhil, ${ }^{3}$ Joon Yau Leong, BS, ${ }^{1}$ Veda N. Giri, MD ${ }^{1,2}$

Affiliation:

1. Department of Urology, Sidney Kimmel Cancer Center, Thomas Jefferson University, Philadelphia, PA, USA

2. Cancer Risk Assessment and Clinical Cancer Genetics, Division of Population Science, Department of Medical Oncology, Sidney Kimmel Cancer Center, Thomas Jefferson University, Philadelphia, Pennsylvania

3. Division of Biostatistics, Department of Pharmacology and Experimental Therapeutics, Sidney Kimmel Medical College, Thomas Jefferson University, Philadelphia, Pennsylvania

Corresponding Author:

Thenappan Chandrasekar, MD

Department of Urology, Thomas Jefferson University

1025 Walnut Street, Suite 1100

Philadelphia PA 19107

United States

Phone: 732-742-1025

Fax: 844-351-9508

Email: thenappan.chandrasekar@gmail.com

Funding Source: None

Conflicts of Interest: All authors report no COI

Article Type/Category: Original

Summary

Abstract: 268

Manuscript: 2531

Tables: 4

Figures: 2

\section{Author Contribution:}

1) TC: Conceptualization, Data Curation, Formal Analysis, Writing (Original, Review \& Editing)

2) SH: Statistical Analysis, Writing (Review \& Editing)

3) JYL, LG: Data Curation, Writing (Review \& Editing)

4) VNG: Conceptualization, Resources \& Supervision, Writing (Review \& Editing)

5) LGG: Resources \& Supervision 


\section{STRUCTURED ABSTRACT}

Background: Prostate cancer (PCa) is increasingly recognized as part of hereditary cancer syndromes (HCS). HCS prevalence among diverse probands seeking genetic evaluation with PCa family history (FHx) has not been reported and has implications for cascade genetic testing (CGT).

Objective: Evaluate rates of HCS among probands reporting PCa FHx and germline mutations among probands. Design, Settings and Participants: Prospective genetic testing database queried for individuals with PCa FHx. Pedigrees analyzed for three HCS: hereditary breast and ovarian cancer [HBOC], Hereditary PCa [HPC], Lynch syndrome [LS].

Outcome Measurements and Statistical analysis: Associations between HCS overall and with plausible link to PCA FHx and race evaluated using Fisher's exact test. Germline mutation rates described among probands with suspicion of HCS connected with PCa FHx.

Results and Limitations: 345 probands reported PCa FHx: 53 African-American (AA), 292 Caucasian (Wh). Overall, 220 probands (63.8\%) met criteria for at least one HCS with potential link to PCa FHx (75.5\% AA; 61.6\% Wh). HBOC linked to PCa FHx was identified in a higher percentage of AA than Wh probands (90.2\% vs. $74.6 \%, p=0.04)$. Among probands who underwent genetic testing with any HCS potentially linked to PCa FHx ( $n=169), 19.5 \%$ had germline mutations identified; 5 AA probands had germline mutations (all in BRCA1/2) while 28 Wh probands had mutations in a spectrum of genes.

Conclusion: A significant percentage of AA probands with PCa FHx meet criteria for HCS, with HBOC identified at the highest rate. Though limited in sample size, our findings implicate BRCA mutations in AA families with HCS linked with PCa, underscoring the need for greater enrollment of AA participants in genetic studies.

Patient Summary: Hereditary cancer syndromes potentially linked to prostate cancer are common in patients reporting a family history of prostate cancer. African-American patients may need special attention with 
regards to testing for hereditary breast and ovarian cancer syndrome which may impact men with prostate cancer in these families.

Keywords: Prostate cancer, genetic testing, hereditary cancer syndrome, lynch syndrome, HBOC, BRCA1, BRCA2

Twitter Statement: @tchandra_uromd @vedangiri @KimmelCancerCtr shed light on race-specific HCS in patients with a family history of \#prostatecancer. 


\section{INTRODUCTION}

Prostate cancer (PCa) has a significant heritable component. ${ }^{1}$ A family history (FHx) of PCa in firstdegree male relatives has been consistently shown to increase the risk of PCa development. ${ }^{1,2}$ Furthermore, PCa has been implicated in multiple hereditary cancer syndromes (HCS) including hereditary PCa (HPC), hereditary breast and ovarian cancer (HBOC), and Lynch syndrome (LS). ${ }^{3-9}$ These expanded insights into the syndromic link of PCa has increased the awareness that PCa can be genetically connected to a spectrum of cancers in families, which is important to inform genetic testing of probands and cascade genetic testing (CGT) of men with PCa in families. ${ }^{3,4}$

Multigene testing has now enabled testing of multiple cancer risks and HCS in probands. ${ }^{10,11}$ Commercial labs offer multigene panels that encompass risks for a spectrum of cancers, and probands seeking hereditary cancer evaluation typically can choose focused cancer testing or large cancer panels based upon personal history, $\mathrm{FHx}$, and patient preference. ${ }^{10} \mathrm{FHx}$ in particular can lead to broader gene testing in probands beyond their presenting cancer risk concern to encompass the cancers reported in families for greater chance of identifying a genetic basis for cancers occurring in a family, inform screening, and guide CGT. Information on PCa FHx is therefore critical to factor in to hereditary cancer discussions with probands, and has not been historically significant prior to recent genetic insights and expanded genetic testing capability for inherited PCa. ${ }^{4,12,13}$ Indeed, multiple genes associated with HBOC, HPC, and LS lend varying degree of risk for inherited PCa and include BRCA1, BRCA2, MLH1, MSH2, PMS2, MSH6, and HOXB13. ${ }^{1,4}$ Furthermore, men with PCa have been shown to carry a spectrum of germline mutations in DNA repair genes, several of which are associated with disease aggressiveness. ${ }^{4,12-16}$ NCCN guidelines have also significantly expanded to address multigene testing for men with PCa with the recognition of the impact on CGT in families. ${ }^{8,9}$ Therefore, intake of PCa FHx from female and male probands is essential to guide extent of genetic testing, uncover cancer risks, and inform CGT for men. 
However, there is currently limited knowledge of the overall prevalence of HCS in families where probands report a PCa FHx, particularly from female and male probands encompassing racial diversity. While prior studies have reported on the impact of PCa FHx on the risk of breast cancer development, ${ }^{17}$ data from cancer genetics programs encompassing a spectrum of patients seeking hereditary cancer assessment are needed to gain deeper insights into the rates of HCS in families reporting a FHx of PCa. Furthermore, there is virtually no data in racially diverse patient populations regarding rates of HCS from probands presenting for genetic assessment who report a PCa FHx. African Americans (AA) in general have been reported to have lower rates of uptake and negative views of genetic testing, ${ }^{18-20}$ which hinders insights into genetic contribution to PCa risk and may limit the benefit of genetic assessment in these families. Expert consensus opinion has stressed the need to engage AA men in genetic evaluation for broader knowledge of PCa predisposition and aggressiveness, ${ }^{4}$ which would be strengthened by knowledge of the link of PCa to HCS in AA families.

Here we report the prevalence of HCS in proband families who reported a FHx of PCa when seeking hereditary cancer assessment at an academic cancer genetics program. We assessed rates of HCS with plausible genetic link to a reported family member with PCa to uncover the magnitude of the need to discuss CGT with men. In particular, we report these rates of HCS by race, which is a current gap in the literature. We furthermore report on the rates of germline mutations identified in probands who underwent genetic testing to explore the genetic spectrum to inform CGT in these families and uncover needs for genetic studies in AA families.

\section{METHODS}


This study included probands from an overall cohort of patients presenting for inherited cancer evaluation and discussed at a Genetics Case Conference as part of the Cancer Risk Assessment and Clinical Cancer Genetics Program at Sidney Kimmel Cancer Center at Thomas Jefferson University. An IRB-approved database including personal history, family history, demographic, race (self-reported) and genetic data has been established (IRB \#16D.743). Probands with self-reported AA or Caucasian (Wh) race were included in this analysis. Pedigrees of probands were constructed as part of their evaluation, and those who reported with a FHx of PCa were selected for this analysis. Criteria for meeting one of three HCS were evaluated based upon proband personal and FHx as follows: HBOC (NCCN Guidelines BRCA1/2 testing criteria, version 2.2017), ${ }^{21}$ Hereditary Prostate Cancer (HPC) (Hopkins Criteria), ${ }^{22}$ or Lynch Syndrome (LS) (Revised Bethesda Criteria). ${ }^{23}$ Plausible genetic link of the family member with PCa to any of the HCS identified in a particular pedigree was determined if the family member with PCa was: (1) a blood relative on the same side of the family meeting HCS criteria (paternal vs. maternal) or (2) a blood relative to the proband if the proband alone met HCS criteria. As per standard cancer genetics practice, probands had undergone genetic counseling with options for genetic testing discussed including focused cancer panel or large cancer panel. ${ }^{18,24}$ If the proband underwent genetic testing, then genetic mutations identified in the proband and/or the family were also collected and reported.

Demographic characteristics of the probands were summarized with counts and percentages or means and standard deviations. Associations between HCS (presence or absence) and race were evaluated using Fisher's exact test. Associations between HCS linked to PCA FHx and race were also evaluated using Fisher's exact test, in the subset of subjects meeting criteria for each HCS. All analyses were performed in SAS 9.4 (SAS Institute Inc., Cary, NC). 


\section{RESULTS}

As of July 1, 2017, 1435 patients had presented for inherited cancer evaluation to the Cancer Risk Assessment and Clinical Cancer Genetics Program and were discussed at the Genetics Case Conference. Among these, 345 probands reported a FHx of PCa ( $84.6 \%$ Wh and $15.4 \%$ AA). Table 1 highlights the demographics of the cohort, and Figure 1 shows the flow of participant data and results for this report. Most probands were female (74.5\%). The most common cancers in probands overall were breast cancer (43.2\%) and PCa (12.8\%). FHx of PCa was reported on the paternal side in $64.9 \%$ of probands, on the maternal side in $34.8 \%$, and within the nuclear family of the proband in $13.0 \%$.

Of the 345 probands reporting a FHx of PCa, 79.4\% $(n=274)$ met criteria for at least one HCS based on their personal cancer history and/or family cancer history (Table 2). The most common HCS pattern was for HBOC (71.3\%), followed by LS (10.4\%), and then HPC (8.4\%). No significant differences were seen in overall or specific HCS rates between AA and Wh pedigrees (83.0\% vs. 78.8\%, respectively, p = 0.58) (Table 2 ).

The potential genetic link of the HCS identified in proband families to the family member with PCa was assessed and shown in Table 3, as well as highlighted by examples in Figure 2. Overall, 220 probands (63.8\% of the entire cohort) met criteria for at least one HCS with a plausible genetic link to the reported family member with PCa, with $75.5 \%$ among AA probands and $61.6 \%$ among Wh probands. Among probands meeting criteria for HBOC, a linked FHx of PCa was identified in a higher percentage of AA families compared to Wh families (90.2\% vs. $74.6 \%$, respectively; $p=0.04)$. While not significant, a similar trend was identified for HCS overall (90.9\% of AA probands vs. $78.3 \%$ of Wh probands; $p=0.06)$. There was no significant difference in having a linked FHx of PCa based on race among probands meeting criteria for HPC or LS (Table 3).

Among probands with a HCS with a plausible genetic link to the family member with PCa who underwent germline testing $(\mathrm{n}=169)$, mutations were identified in $19.5 \%$, with $B R C A 1 / 2$ mutations most commonly identified (10.1\%), followed by mutations in CHEK2 (4.7\%), PALB2 (1.2\%), MSH2 (1.2), APC (0.6\%), 
$\operatorname{ATM}(0.6 \%)$, MUTYH (0.6\%), and PMS2 (0.6\%)(Table 4). While BRCA1/2 mutations accounted for all mutations identified in AA probands $(\underline{n=5)}$, a spectrum of mutations were identified in Wh probands $\underline{(n=28)}$ including in CHEK2, MSH2, PALB2, APC, ATM, MUTYH, and PMS2 (Table 4).

\section{DISCUSSION}

Genetic evaluation for hereditary cancer risk involves factoring in family cancer history, personal cancer history, and germline genetic testing of male and female probands to inform cancer screening, risk reduction, and precision medicine recommendations for probands. ${ }^{4,10,24}$ However, there are broader implications of hereditary cancer assessment for family members regarding CGT if genetic mutations are identified in probands. With the growing recognition of the connection of PCa with specific HCS, ${ }^{1,3,4}$ men with PCa in families meeting HCS criteria can benefit from appropriate CGT when mutations are identified in probands as well as family history-based recommendations. However, there is a lack of data regarding the prevalence of potential PCa connection to HCS in diverse families and the mutation spectrum in probands that may inform CGT for men with PCa. These data are particularly lacking in AA families, where uncovering the prevalence of HCS and germline mutations will lend support to developing strategies to increase awareness of genetic evaluation among AAs.

Our study in a comprehensive academic cancer genetics program found that a significant percentage of probands (63.8\%) who presented for inherited cancer assessment with a FHx of PCa met criteria for a hereditary cancer syndrome where the family member with PCa may have had a plausible genetic link to the HCS in the family. Of further interest, HBOC was identified in a higher percentage of AA families with a potentially linked PCa compared to Wh families $(p=0.04)$. This is the first report to our knowledge to show that AA probands with a FHx of PCa have a higher chance of meeting criteria for HBOC, with implications for proband genetic testing, downstream CGT of AA men with PCa, and family history-based recommendations for 
men. Even if probands test negative, men with PCa in these families with suspected HCS may be recommended to undergo their own genetic testing. ${ }^{24}$ Family history-based recommendations are also important for all blood relatives particularly when there is a suspected HCS with uninformative genetic testing. ${ }^{4,8,24}$ Therefore, men with PCa in families where probands may present for cancer concerns even other than PCa can be benefited by family history-based recommendations for cancer screening or pursue their own genetic testing. ${ }^{24}$

Though our sample size was small, our findings show that BRCA1/2 mutations accounted for all mutations identified in AA probands while a spectrum of mutations were identified in Wh probands including in $C H E K 2, M S H 2, P A L B 2, A P C, A T M, M U T Y H$, and PMS2. Our findings point to the need for greater engagement of AA families in genetic evaluation to broaden our insights into the genetic predisposition to cancers affecting females and males in families. Germline mutations in families reporting a FHx of PCa further highlight the need to develop strategies to address communication of cancer genetic evaluation across genders, with particular focus needed in AA families. CGT of men with PCa has major emerging implications for precision therapy. Olaparib was given "Breakthrough Therapy" designation by the United States FDA for BRCA1/2- or ATM-positive metastatic castration-resistant PCa (mCRPC) based on Phase II data from the TOPARP-A trial demonstrating improved responses particularly in a subset of patients with DNA repair mutations. ${ }^{25}$ Recently, rucaparib was also granted "Breakthrough Therapy" designation for men with BRCA1/2-positive mCRPC following at least one androgen receptor-directed therapy and taxane-based chemotherapy based on Phase II data from the TRITON2 study. ${ }^{26}$ Furthermore, BRCA2 status may inform treatment sequencing in men with mCRPC. ${ }^{27}$ BRCA and ATM mutations are also beginning to inform active surveillance in men with early stage PCa. ${ }^{28}$ Therefore, identification of men with PCa in families with suspected HCS and germline mutations can inform precision therapy or management for men with PCa which again needs to be specifically addressed in AA families as our data highlight. Genetic results inform additional cancer risks in families, where guidelines 
for cancer screening exist for several genes. For example, cancer risks associated with BRCA mutations include PCa, pancreatic cancer, male breast cancer, and melanoma for men. Female cancer risks include breast cancer, ovarian cancer, pancreatic cancer, and melanoma which all warrant discussion of cancer screening, high-risk consultation, and/or risk reducing recommendations. ${ }^{8}$ These discussions need special attention in AA families to ensure appropriate CGT of men with PCa and other blood relatives to inform cancer screening and cancer risk reduction measures.

A spectrum of germline mutations was observed in Wh probands beyond $B R C A$, which has implications for CGT in genetically-linked men with PCa in these families. In addition to the cancer risks associated with BRCA mutations, pathogenic variants in CHEK2, MSH2, PALB2, APC, ATM, MUTYH, and PMS2 confer a host of cancer risks for male carriers including colorectal cancer, pancreatic cancer, and small bowel cancer which may be relevant for men with PCa who are in survivorship for heightened cancer screening. ${ }^{8,10,24}$ For men with mCRPC, genetic results from CGT may inform therapeutic options for PARP inhibition and also for immunotherapy. ${ }^{25,29}$

There are some limitations to note. The population in this analysis was enriched for patients with a high likelihood of HCS, as all patients were specifically referred for genetic testing and therefore the rates of HCS may be higher than the general population. Additionally, despite the fact that the referral base for this study is a population with a higher AA population than other parts of the country, AA probands represented $15.4 \%$ of this study, which highlights the need to engage AAs in genetic evaluation discussions and could have inflated the rates of HCS in AA participants in our study. Family history information was by proband selfreport, which is a known limitation in cancer genetic studies as well. ${ }^{30}$ Self-reported FHx was also limited regarding information of clinically significant PCa, which is a part of NCCN guidelines informing genetic testing..$^{8,9}$ Genetic testing in our cohort encompassed focused gene testing and large panel testing based on patient decisions after genetic counseling, which may have influenced our findings. The overall number of AA 
probands who underwent genetic testing was also limited $(n=28)$, of whom five were found to carry $B R C A 1 / 2$ mutations. While this implicates BRCA mutations in AA families with HCS linked with PCA, there remains a need for broader testing to uncover the genetic spectrum in AA families. A strength of our study is the large percentage of female probands included in the analysis, which begins to address the gender divide regarding cancer genetic testing and communication of genetic results in families with attention to diversity.

\section{CONCLUSION}

A significant percentage of patients who present for inherited cancer assessment with a FHx of PCa meet criteria for a HCS with a plausible genetic link to a family member with PCa. AA probands with a PCa FHx have substantial rates of HCS with a subset having BRCA mutations identified, thus having CGT implications for men with PCa in these families. Cascade testing for men with PCa in families presenting with a broad spectrum of cancers is crucial to inform cancer screening, precision therapy, and precision management of men with PCa. Greater research is needed to optimize communication of cancer risk and genetic testing across genders with attention to patient diversity.

ACKNOWLEDGEMENTS: None. The authors express appreciation to the patients and their families who participated in this study. 


\section{REFERENCES / BIBLIOGRAPHY}

1. Genetics of Prostate Cancer (PDQ $\left.{ }^{\circledR}\right)$. National Cancer Institute. [Accessed May 2, 2019]. Available at:

https://www.cancer.gov/types/prostate/hp/prostate-genetics-pdq

2. Kicinski M, Vangronsveld J, Nawrot TS. An epidemiological reappraisal of the familial aggregation of prostate cancer: A meta-analysis. PLoS One. 2011;6(10):e27130. doi: 10.1371/journal.pone.0027130 [doi].

3. Giri VN, Beebe-Dimmer JL. Familial prostate cancer. Semin Oncol. 2016;43(5):560-565. doi: S0093-7754(16)30060-4 [pii].

4. Giri VN, Knudsen KE, Kelly WK, et al. Role of genetic testing for inherited prostate cancer risk: Philadelphia prostate cancer consensus conference 2017. J Clin Oncol. 2018;36(4):414-424. doi: 10.1200/JCO.2017.74.1173 [doi].

5. Mersch J, Jackson MA, Park M, et al. Cancers associated with BRCA1 and BRCA2 mutations other than breast and ovarian. Cancer. 2015;121(2):269-275. doi: 10.1002/cncr.29041 [doi].

6. Haraldsdottir S, Hampel H, Wei L, et al. Prostate cancer incidence in males with lynch syndrome. Genet Med. 2014;16(7):553-557. doi: 10.1038/gim.2013.193 [doi].

7. Raymond VM, Mukherjee B, Wang F, et al. Elevated risk of prostate cancer among men with lynch syndrome. J Clin Oncol. 2013;31(14):1713-1718. doi: 10.1200/JCO.2012.44.1238 [doi].

8. NCCN clinical practice guidelines in oncology (NCCN guidelines ${ }^{\circledR}$ ): Genetic/familial high-risk assessment: Breast and ovarian (version 3.2019). https://www.nccn.org/professionals/physician gls/pdf/genetics screening.pdf. Updated 2019. Accessed February 19, 2019.

9. NCCN clinical practice guidelines in oncology (NCCN guidelines ${ }^{\circledR}$ ): Prostate (version 1.2019). https://www.nccn.org/professionals/physician gls/pdf/prostate.pdf. Updated 2019. Accessed April 26, 2019. 
10. Hall MJ, Forman AD, Pilarski R, Wiesner G, Giri VN. Gene panel testing for inherited cancer risk. J Natl Compr Canc Netw. 2014;12(9):1339-1346. doi: 12/9/1339 [pii].

11. Robson ME, Bradbury AR, Arun B, et al. American society of clinical oncology policy statement update: Genetic and genomic testing for cancer susceptibility. J Clin Oncol. 2015;33(31):3660-3667. doi: 10.1200/JCO.2015.63.0996 [doi].

12. Pritchard CC, Mateo J, Walsh MF, et al. Inherited DNA-repair gene mutations in men with metastatic prostate cancer. N Engl J Med. 2016;375(5):443-453. doi: 10.1056/NEJMoa1603144 [doi].

13. Nicolosi P, Ledet E, Yang S, et al. Prevalence of germline variants in prostate cancer and implications for current genetic testing guidelines. JAMA Oncol. 2019. doi: 10.1001/jamaoncol.2018.6760 [doi].

14. Narod SA, Neuhausen S, Vichodez G, et al. Rapid progression of prostate cancer in men with a BRCA2 mutation. Br J Cancer. 2008;99(2):371-374. doi: 10.1038/sj.bjc.6604453 [doi].

15. Castro E, Goh C, Leongamornlert D, et al. Effect of BRCA mutations on metastatic relapse and cause-specific survival after radical treatment for localised prostate cancer. Eur Urol. 2015;68(2):186-193. doi: 10.1016/j.eururo.2014.10.022 [doi].

16. Na R, Zheng SL, Han M, et al. Germline mutations in ATM and BRCA1/2 distinguish risk for lethal and indolent prostate cancer and are associated with early age at death. Eur Urol. 2017;71(5):740-747. doi: S0302-2838(16)30885-5 [pii].

17. Beebe-Dimmer JL, Yee C, Cote ML, et al. Familial clustering of breast and prostate cancer and risk of postmenopausal breast cancer in the women's health initiative study. Cancer. 2015;121(8):1265-1272. doi: 10.1002/cncr.29075 [doi].

18. Hall MJ, Olopade OI. Disparities in genetic testing: Thinking outside the BRCA box. J Clin Oncol. 2006;24(14):21972203. doi: 24/14/2197 [pii]. 
19. Hann KEJ, Freeman M, Fraser L, et al. Awareness, knowledge, perceptions, and attitudes towards genetic testing for cancer risk among ethnic minority groups: A systematic review. BMC Public Health. 2017;17(1):8. doi: 10.1186/s12889017-4375-8 [doi].

20. Sheppard VB, Graves KD, Christopher J, Hurtado-de-Mendoza A, Talley C, Williams KP. African american women's limited knowledge and experiences with genetic counseling for hereditary breast cancer. J Genet Couns. 2014;23(3):311322. doi: 10.1007/s10897-013-9663-6 [doi].

21. Daly MB, Pilarski R, Berry M, et al. NCCN guidelines insights: Genetic/familial high-risk assessment: Breast and ovarian, version 2.2017. J Nat/ Compr Canc Netw. 2017;15(1):9-20. doi: 15/1/9 [pii].

22. Carter BS, Bova GS, Beaty TH, et al. Hereditary prostate cancer: Epidemiologic and clinical features. J Urol. 1993;150(3):797-802. doi: S0022-5347(17)35617-3 [pii].

23. Umar A, Boland CR, Terdiman JP, et al. Revised bethesda guidelines for hereditary nonpolyposis colorectal cancer (lynch syndrome) and microsatellite instability. J Natl Cancer Inst. 2004;96(4):261-268.

24. Riley BD, Culver JO, Skrzynia C, et al. Essential elements of genetic cancer risk assessment, counseling, and testing: Updated recommendations of the national society of genetic counselors. J Genet Couns. 2012;21(2):151-161. doi: 10.1007/s10897-011-9462-x [doi].

25. Mateo J, Carreira S, Sandhu S, et al. DNA-repair defects and olaparib in metastatic prostate cancer. N Engl J Med. 2015;373(18):1697-1708. doi: 10.1056/NEJMoa1506859 [doi].

26. Clovis oncology receives breakthrough therapy designation for rubraca ${ }^{\circledR}$ (rucaparib) for treatment of BRCA1/2mutated metastatic castration resistant prostate cancer (mCRPC). https://ir.clovisoncology.com/investors-andnews/news-releases/press-release-details/2018/Clovis-Oncology-Receives-Breakthrough-Therapy-Designation-forRubraca-rucaparib-for-Treatment-of-BRCA12-Mutated-Metastatic-Castration-Resistant-Prostate-CancermCRPC/default.aspx. Accessed Feb 18, 2019. 
27. Castro E, Romero-Laorden N, Del Pozo A, et al. PROREPAIR-B: A prospective cohort study of the impact of germline DNA repair mutations on the outcomes of patients with metastatic castration-resistant prostate cancer. J Clin Oncol. 2019;37(6):490-503. doi: 10.1200/JCO.18.00358 [doi].

28. Carter HB, Helfand B, Mamawala M, et al. Germline mutations in ATM and BRCA1/2 are associated with grade reclassification in men on active surveillance for prostate cancer. Eur Urol. 2018. doi: S0302-2838(18)30684-5 [pii].

29. Carlo MI, Giri VN, Paller CJ, et al. Evolving intersection between inherited cancer genetics and therapeutic clinical trials in prostate cancer: A white paper from the germline genetics working group of the prostate cancer clinical trials consortium. JCO Precis Oncol. 2018;2018:10.1200/PO.18.00060. Epub 2018 Aug 16. doi: 10.1200/PO.18.00060 [doi] 30. Kerber RA, Slattery ML. Comparison of self-reported and database-linked family history of cancer data in a casecontrol study. Am J Epidemiol. 1997;146(3):244-248. 